\title{
Role of Wireless Communication in Healthcare System to Cater Disaster Situations Under 6G Vision
}

\author{
Muhammad Bilal Janjua ${ }^{1}$, Ahmet Enes Duranay ${ }^{1 *}$ and Hüseyin Arslan ${ }^{1,2}$ \\ ${ }^{1}$ Department of Electrical and Electronics Engineering, Istanbul Medipol University, Istanbul, Turkey, ${ }^{2}$ Department of Electrical \\ Engineering, University of South Florida, Tampa, FL, United States
}

OPEN ACCESS

Edited by:

Hina Tabassum

York University, Canada

Reviewed by:

Nasir Saeed,

King Abdullah University of Science and Technology, Saudi Arabia

Faheem Ahmad Khan,

University of Huddersfield,

United Kingdom

*Correspondence:

Ahmet Enes Duranay

ahmet.duranay@std.medipol.edu.tr

Specialty section:

This article was submitted to

Wireless Communications,

a section of the journal

Frontiers in Communications and

Networks

Received: 27 September 2020 Accepted: 10 November 2020

Published: 15 December 2020

Citation:

Janjua MB, Duranay $A E$ and Arslan $H$ (2020) Role of Wireless

Communication in Healthcare System to Cater Disaster Situations Under 6G Vision. Front. Comms. Net. 1:610879

doi: 10.3389/frcmn.2020.610879
The natural disasters created by infectious diseases have a formidable impact on people and societies. Without affecting the city infrastructure, pandemics leave the places abandoned because of the shortage of human resources, either due to deaths, illness, or unwillingness to work because of health risks. However, providing a timely response can prevent losses from occurring due to the virus dissemination. Since the first reported case of influenza in 1918 to the current pandemic Coronavirus Disease-2019 (COVID-19), the system playing a key role in saving human lives is healthcare. Nowadays, smart healthcare system development is a popular trend and wireless communication is the backbone of such systems. To provide patients with diagnosis, treatments, and several health services both within hospitals and remotely, all the healthcare units must be equipped with advanced technologies. A rapid response unit is also required to handle the thrust of the patients and queries generated during disasters. This paper discusses healthcare communication challenges and possible solutions for early awareness and rapid response in disaster situations under the human-centric vision of sixth-generation wireless technologies.

Keywords: disaster management, COVID-19, rapid response, healthcare communication system, $6 \mathrm{G}$ vision

\section{INTRODUCTION}

Since the beginning of the twentieth century, an increase in natural disasters has been witnessed. These can be hurricanes, earthquakes, tsunamis, nuclear accidents or pandemics. Each left a dreadful impact on human societies, particularly in terms of life loss, health problems, loss of infrastructure or economic collapse (Coronese et al., 2019). Even though great technological advancements have been made over the last few years, dealing with such incidents is still not an easy task, especially the ones directly endangering human health, like the recent pandemic i.e., Coronavirus Disease-2019 (COVID-19). A large number of people passed away in the last few months, in part due to the lack of required medical care, following the declaration of the pandemic because the healthcare infrastructure was not designed considering catastrophic scenarios. COVID-19 manifested that the surge of patients, lack of medical facilities, failure of infection prevention, and delayed response can collapse the healthcare infrastructure even in most developed countries in the world (Chamola et al., 2020). Having a smart healthcare system that ensures rapid and safe health services for patients and healthcare providers within the hospitals and in remote areas can help in minimizing the fallout due to disasters (Cook et al., 2018; Fieux et al., 2020; Willan et al., 2020). Therefore, special efforts are required regarding improvement and development of a healthcare system that can operate efficiently under these situations. 
The current healthcare system relies heavily on the fourth-generation (4G) networks to enable smart healthcare applications; however, due to limited bandwidth resources, these networks cannot support the rapid growth of these applications in the long run. Compared with $4 \mathrm{G}$, fifth-generation (5G) networks offer better services in terms of bandwidth, reliability, latency, and data rate (Li, 2019). 5G services are broadly categorized into enhanced mobile broadband (eMBB), ultra reliable low latency communications (URLLC), and massive machine type communications (mMTC). From the healthcare system perspective, $5 \mathrm{G}$ services: (i) provide internet connectivity to the internet of medical things (IoMT) devices through mMTC; (ii) offer high quality video calling for telemedicine and augmented reality (AR)/virtual reality (VR) for better visualization in diagnosis and treatment through eMBB; (iii) support drones and autonomous vehicles for both the surveillance and emergency scenarios with URLLC (Ahad et al., 2019). Nevertheless, the services offered by $5 \mathrm{G}$ are insufficient to satisfy the evolution of healthcare technologies as well as for the disaster conditions. In particular, privacy and security for remote healthcare, ubiquitous communication for telemedicine in areas with insufficient infrastructure (either due to digital divide or disaster scenarios), connectivity for ultra-dense IoMT devices and extremely reliable and low latency communication for both the remote surgery using mixed reality.

Nowadays, sixth-generation (6G) networks and their application scenarios are under discussion. One key motivation is to develop the communication from a society and humanitarian perspective i.e., designing the communication technologies to improve the quality of life in all aspects (Da Costa Daniel Benevides, 2020; Dang et al., 2020; MatinmikkoBlue et al., 2020; Rahman et al., 2020). In this regard, many services and technologies are being proposed under the 6G vision in the literature, such as holographic telepresence, tactile internet, autonomous and tele-operated vehicles, aerial networks, internet of bio-nano things (IoBNT), uncoordinated networks and co-existence of radio frequency (RF), visible light communication (VLC), and Terahertz (THz) (Akyildiz et al., 2020). These technologies would help in developing a strong communication infrastructure for the smart healthcare system in terms of extreme reliability, ultra massive connectivity, connection continuity, network scalability, and flexibility for rapid response, allowing monitoring and taking preventive actions in pandemic situations. Physical layer security is another significant technology especially for e-health applications, which is expected to be offered in $6 \mathrm{G}$ for making the communication link secure (Vora et al., 2018). Moreover, in case of natural disasters, information on population activities, such as mobility, can give insight into the scale and location of the damage.

Recently, some research studies have been conducted on COVID-19 (Chamola et al., 2020; Rahman et al., 2020; Saeed et al., 2020; Wijesooriya et al., 2020). Telehealth as a potential way to provide better healthcare and educate the medical staff during COVID-19 is proposed in Wijesooriya et al. (2020). COVID-19 management and analysis solutions deploying the deep learning algorithms using edge computing technology in the beyond 5G framework is discussed in Rahman et al. (2020).
The impact of the COVID-19 outbreak on human living and the use of $5 \mathrm{G}$ technologies along with IoT, drones, etc., to manage the situation are comprehensively discussed in Chamola et al. (2020). The importance of wireless communication technologies in combating the global economic challenges due to COVID-19 is highlighted in Saeed et al. (2020). Particularly, in monitoring the virus dissemination, improving the health, education, and economic sectors. Additionally, some challenges faced by wireless communication related to privacy and security are also discussed. Although the literature has presented several technological solutions to manage the situation created by the COVID19 outbreak yet there is a need to discuss challenges faced by the healthcare system during disasters and to overcome these through the technological advancements envisioned for $6 \mathrm{G}$ Networks.

This perspective article presents the role of wireless communication in the healthcare system during regular and pandemic situations. We first categorize the healthcare system into three areas, hospital, remote healthcare, and disaster response unit. Then, we investigate the problems of healthcare communication system in each area along with the possible solutions, and present a vision of $6 \mathrm{G}$ from the healthcare perspective. We believe that the healthcare system with the inclusion of $6 \mathrm{G}$ technologies can help in handling the pandemic situations more effectively and minimize the fatalities.

The article is organized as follows. Section 2 summarizes the healthcare system applications and challenges. Solutions for healthcare communications are proposed in section 3 and critical discussions are provided in the end.

\section{HEALTHCARE SYSTEM APPLICATIONS AND CHALLENGES}

Getting better healthcare from diagnosis to treatments is a basic human need. High quality healthcare must be available for everyone and everywhere, which is now possible with the development of smart healthcare applications. In this paper, we categorize the healthcare system into three major application areas; hospital environment, remote healthcare, and disaster response unit as shown in Figure 1.

\subsection{Hospital Environment}

A hospital consists of professionally trained staff and medical devices for providing healthcare to the people in the best possible way. In order to run the hospital operations smoothly, efficient communication between administration, doctors, medical staff, and patients is required. Commonly used technologies for interdisciplinary communication within the hospital are email, mobile phone, pagers and two-way radios. Recently, new methods are also being used to improve the healthcare services in the hospital; collecting and maintaining the health records electronically help in reducing human errors, saves time and improves communication between physicians and patients, introducing artificial intelligence (AI) to make the better decisions in diagnosis, involving robots to achieve high precision in surgery as well as supporting the medical staff, and IoMT allows the 


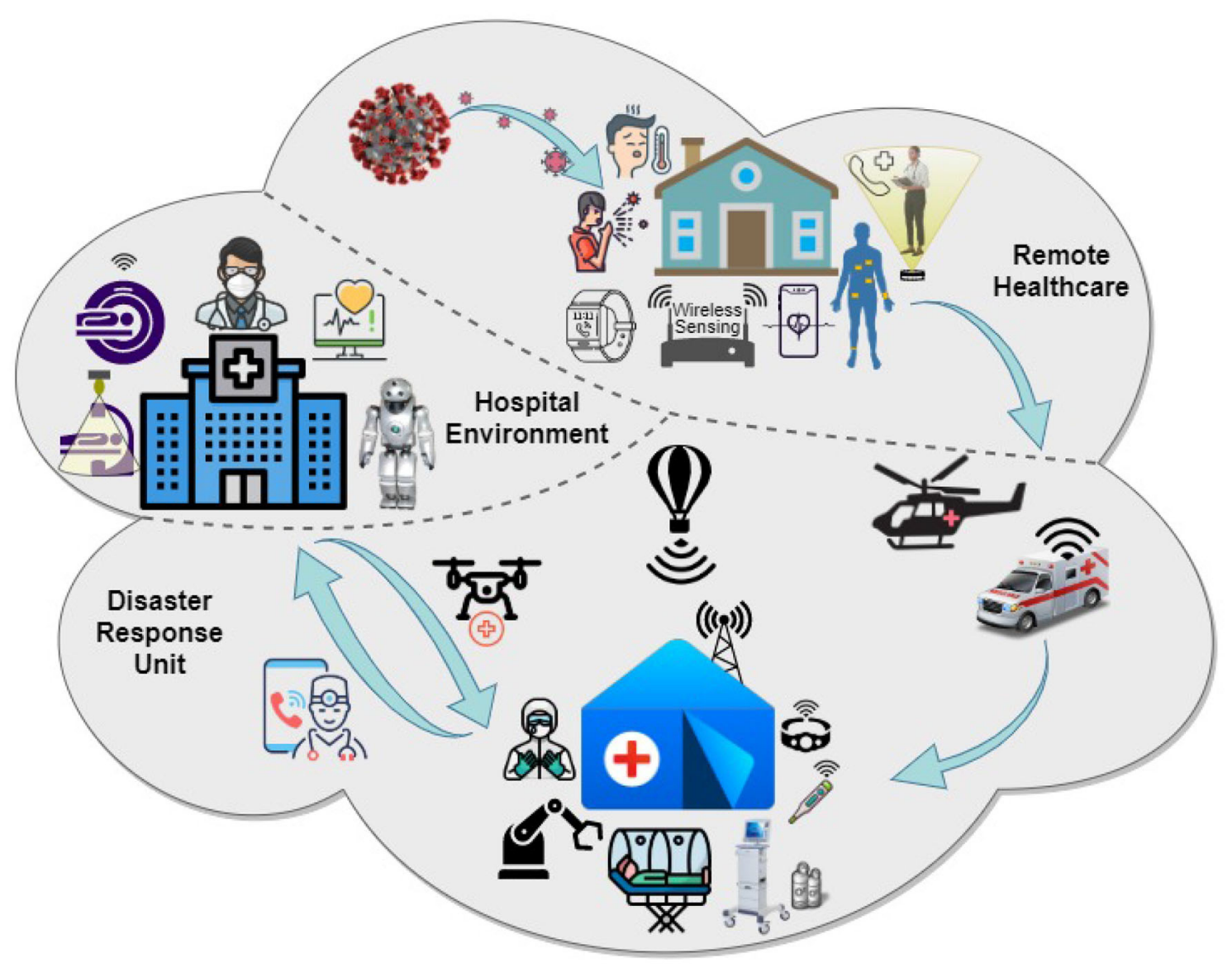

FIGURE 1 | Healthcare system categorization for pandemic response.

wearable devices and sensors to send data directly to the medical data collection center (Boyle, 2006; Tian et al., 2019).

In disaster scenarios, hospitals become overwhelmed quickly with the large number of patients admitted to the hospital. The shortage of medical staff and equipment as well as limited patient capacity makes it difficult to control the situation. Particularly, in pandemic situations, things become worse because the infectious disease can be transferred from patients to medical staff, thus, ensuring the safety of staff during healthcare provision to infected patients becomes the biggest challenge. One way to overcome this challenge is to minimize the physical interaction between patients and clinicians. This can be achieved by deploying IoMT and robots in the hospital environment (Chamola et al., 2020). IoMT wearable devices and sensors can monitor and transmit the patients health records to the clinicians over the internet resulting in reduced interactions (Hassija et al., 2019). On the other hand, robots can be used in many ways such as delivering supplies, medical checkups and disinfecting the patients' area. Use of these devices and robots at massive scale is expected in the near future to provide safety and support to the medical staff and doctors at the hospital.

All these technological advancements rely on the communication infrastructure of the hospital and require a secure, reliable and high data rate communication link. RF based wireless communication i.e., Wi-Fi network has been widely deployed in hospitals to enable communication with better capacity, mobility, positioning and localization (Ahmed et al., 2020). However, the medical equipment is prone to electromagnetic interference (EMI), which restricts the use of $\mathrm{Wi}-\mathrm{Fi}$ networks. Likewise, the complex physical structure of hospital limits penetration of highfrequency signals through walls and creates coverage issues. Additionally, existing Wi-Fi networks were not deployed from the perspective of accommodating the traffic load in disaster situations. Thus, during high patient load, a Wi-Fi network is insufficient to fulfill the communication requirements in terms of capacity and connectivity. Furthermore, real-time monitoring of health records through IoMT devices require massive connectivity, high reliability and low latency. A small delay and poor connectivity may cause severe consequences in case of emergencies. Extremely reliable and low latency communication is necessary in hospitals, particularly to enable the use of robot technology. It is also crucial to protect the communication link through advance security techniques to prevent the patients' privacy and critical information from unauthorized access. 


\subsection{Remote Healthcare}

Remote healthcare is globally prevalent under current situations for providing healthcare to people living in rural areas and those with travel limitations. This service is facilitated in two ways; telemedicine and remote health monitoring (Pramanik et al., 2019). In telemedicine, physicians use teleconferencing or check electronic health records for evaluation, diagnosis and treatment of patients without physical interaction. It can be availed from home or basic health units under the supervision of a paramedic. Currently, high quality video teleconferencing is used for telemedicine; however, with the rapid development of hologram technology, holographic telepresentation, is expected to be used in the near future. For remote health monitoring, IoMT technologies such as wearable devices and wireless body area network (WBAN) have started to be deployed extensively (Wei et al., 2020). Likewise, implementation of real-time monitoring systems helps to improve the elderly people's living by tracking emergency situations via sensors and providing urgent care. However, new technology adoption, especially for elderly people, is a big hindrance in achieving this goal. Furthermore, the dose adjustment through digital medicine makes life easier for patients who need regular care, for instance, diabetics. These technologies require a secure and reliable link to ensure the privacy and safety of the patient.

With the emergence of COVID-19, remote healthcare is being promoted to reduce infection spread and patient load at the hospital (Ohannessian et al., 2020). Remote healthcare services completely rely on communication infrastructure. Hence, several applications have not been explored yet due to communication challenges such as massive connectivity and interference for IoMT, ultra-low power communication for biosensors, reliability and low latency for tactile-internet based remote surgery and digital medicine, and high data rate requirements for holographic telepresence. In the aforementioned challenges, securing and protecting the patients critical information and privacy are major ones. The wireless communication link is vulnerable to security threats such as eavesdropping, jamming and spoofing, especially in remote healthcare, where all the devices and sensors send the data to the medical staff in the hospital or upload in the online portal. If the communication link is not secure, an eavesdropper can get the critical information of the patient or a spoofer can manipulate the data in the online database, which can lead to fatalities. Therefore, there is a need to make the physical channel secure in addition to cryptography based techniques.

\subsection{Disaster Response Unit}

Disaster response unit is a system that is required during the response to any hazardous or emergency situation to provide the healthcare services. It includes disaster management centers, emergency mobile service (EMS), field hospitals, monitoring public spaces, etc. When a catastrophic incident happens, a disaster management center is established within a hospital or in a separate institution for rapid response, health services, communication between departments and monitoring the aftereffects. EMS is one of the most critical services of disaster response unit to provide rapid response. In case of any emergency situation outside the hospital, it provides prehospital healthcare, either through the ground or air ambulance, depending on the severity of the emergency situations. Smart ambulances contain sophisticated medical equipment to improve the speed of diagnosis and treatment by taking precautionary measures, updating the medical staff through high quality video calling and immediate remote consultation through the cellular network. It can also reduce the number of patients taken to the hospital by providing the healthcare through telemedicine. Furthermore, autonomous ambulances have been introduced with advanced technologies in some countries for providing the rapid response and saving the paramedics from getting affected (Zarkeshev and Csiszár, 2019). Moreover, the disaster response unit needs to communicate with other EMS linked departments i.e., fire, police, insurance, etc., which requires strong communication and coordination for centralized control. During pandemics, it is critical to inform the EMS staff to take the necessary precautions before providing healthcare to the patients to prevent themselves from being affected by the disease. Regarding the pandemic challenges, infection prevention from spreading is the key issue by maintaining a distance between people. Therefore, along with the EMS, monitoring the public to ensure that they are adhering to pandemic precautions is critical to detect violations and take the necessary measures to prevent the dissemination of disease. Similarly, thermal imaging can be utilized to detect outbreaks. These tasks, along with outdoor disinfection, can be realized with drone deployment from the disaster management center. In order to develop a rapid response system, the availability of a reliable communication system is compulsory.

When a natural disaster, especially the pandemics occur, healthcare systems are hit hardest due to the additional flux of patients. Field hospitals can be vital in these situations to isolate those affected by the pandemic, freeing up other hospitals and allowing better management of limited hospital resources such as equipment. Currently, these hospitals are used to quarantine infected patients and provide proper care. In particular, robot and drone technologies are paving the way to minimize the interaction between the clinicians and patients. These technologies are being used to perform several tasks, such as delivering the food and medical kits, taking test samples, disinfecting the patient areas and so on. Monitoring the patient's health critical signs can be carried through IoMT based smart medical devices i.e., smart thermometers, smart wristbands (Berardinelli et al., 2020). Furthermore, city hospital clinicians can provide consultation to the patients in the field hospital through remote healthcare and telemedicine services (Yaacoub and Alouini, 2020).

From the communication perspective, in a disaster response unit several challenges have been highlighted as follows. In case of autonomous ambulance and delivery drones, high mobility results in a large number of handovers, consequently increasing the latency and decreasing the reliability of the communication link. In order to enable safe driving of these vehicles, reliable vehicle-to-everything (V2X) communication is challenging. Furthermore, in smart ambulances, the realtime transfer of information collected from medical imaging requires high data rate transmission. Likewise, field hospitals usually lack network connectivity due to limited or unavailable 
communication networks. Concerning the security attacks, signals used for dedicated short range communication in autonomous ambulances can be manipulated or controlled for jamming the radar or making collisions. In addition, smart wristbands are used on quarantined patients to monitor and track their locations. However, communication signals of wristbands are vulnerable to jamming and spoofing attacks.

\section{SOLUTIONS AND EMERGING TECHNOLOGIES UNDER 6G VISION}

This section presents solutions to the aforementioned challenges of the healthcare system engaged communication problems along with the discussion on $6 \mathrm{G}$ vision and technologies. Figure 2 presents the deployment of $6 \mathrm{G}$ technologies in different areas of healthcare system to overcome the communication challenges.

\subsection{G Vision and Technologies}

$5 \mathrm{G}$ networks are going to offer better services with significant advancement compared to earlier generations. However, there is a need to fill the gaps of security, intelligence, wireless connectivity, energy efficiency, etc., which require the development of new wireless generation networks. Therefore, $6 \mathrm{G}$ vision is to utilize communication technologies with intelligence for transforming the world into a secure and fully connected digital society. Aggressive research regarding 6G vision categorizes its enabling technologies into (i) large spectrum bandwidth i.e., VLC and THz; (ii) integrated terrestrial-aerial networks i.e., unmanned aerial vehicle (UAV), high-altitude platforms (HAPs), and satellites; (iii) energy efficient and communication environment intelligence i.e., ambient backscatter communication $(\mathrm{AmBC})$, reconfigurable intelligent surface (RIS); (iv) holistic security paradigm i.e., physical layer security (PLS) and blockchain (BC).

Due to the spectrum scarcity in lower frequency bands, sub- $\mathrm{THz}$ bands are proposed to provide the communication with very high bandwidth of $6 \mathrm{G}$ networks. Additionally, other spectrum resources such as VLC are expected to be used as a potential candidate to support the RF spectrum that utilizes commodity lighting infrastructure for high data rate wireless communication, positioning and localization, especially in the indoor environment. Future wireless networks will not be limited to terrestrial networks, rather they will be expanding to cover the space. Integrated terrestrial-aerial communication networks with the UAV and other non-terrestrial networks (NTN) platforms are proposed to reduce the digital divide and provide wireless connectivity everywhere with lower path-loss and higher line of sight (LOS) probability, and flexibility with the mobility advantages (Zhou et al., 2020). Energy efficiency is critical for the internet of things (IoT) devices; thus, AmBC provides ultra low power communication using ambient signals generated from conventional RF systems i.e., FM, TV, WiFi, etc. (Van Huynh et al., 2018). RIS is a passive or semi-passive surface, containing antenna elements to provide an intelligent communication environment, along with improved coverage and capacity via customizing radio signals intelligently before reflection (Akyildiz et al., 2020). Apart from cryptography-based security, $6 \mathrm{G}$ will provide a new solution to the security paradigm with the inclusion of PLS and quantum cryptography. In addition to security, the privacy of the confidential data can be maintained using $\mathrm{BC}$ algorithms. These techniques will solve communication security and users' privacy challenges of the future systems (Hamamreh et al., 2019; Nawaz et al., 2019; Tanwar et al., 2020).

\subsection{Potential Solutions to the Communication Challenges}

The major problems related to communication occur in different areas of the healthcare system include capacity, coverage, connectivity, reliable and low latency communication, sensing, security and privacy. This section presents the solutions for the aforementioned challenges to improve the healthcare system incorporating the $6 \mathrm{G}$ technologies.

\subsubsection{Capacity Enhancement}

After the first wave of COVID-19, network operators are focusing on the planning and development of network infrastructure for disaster scenarios, where capacity enhancement is one of the biggest challenges. For example, a network operator in Turkey has recorded an increase in data usage up to $35 \%$ since the beginning of COVID-19 (Turkcell, 2020). This increase is also prevalent in healthcare systems as a result of higher patient loads and large-scale use of IoMT devices. In order to serve patients and visitors, both within the hospital and remotely, current RF communication infrastructure based on low frequency bands is insufficient. Therefore, the utilization of high-frequency bands and ultra-massive multiple-input multiple-output (MIMO) should be implemented to improve the overall capacity of the network. Alternatively, indoor capacity enhancement can be realized through VLC (Janjua et al., 2020). This technology can complement the existing RF based communication network by sharing the user load as shown in Figure 3C. The ultra high bandwidth of $\mathrm{THz}$ can also support very high data rates in holographic telepresence during remote healthcare (Elayan et al., 2020). In addition, aerial networks such as HAPs can be utilized for network scalability to increase the capacity of the healthcare communication system for field hospitals. RIS integrated UAVs can also be used to increase capacity during the disaster time when additional capacity is required for the hospitals. Having the ability of panoramic full-angle reflection of RIS integrated UAV allows free mobility and reflection of the signals in the air toward the hospital to increase capacity by taking available channels from the neighboring cells. Cognitive radio with AmBC technology also known as symbiotic radio can support these devices, which means the IoMT devices can take the advantage of both the cognitive radios and $\mathrm{AmBC}$ for spectrum sharing and ultra-low power communication, respectively. In this way, connectivity enhancement and energy efficiency can be achieved, simultaneously. However, maintaining the quality of service (QoS) is challenging in these technologies (Van Huynh et al., 2018). 


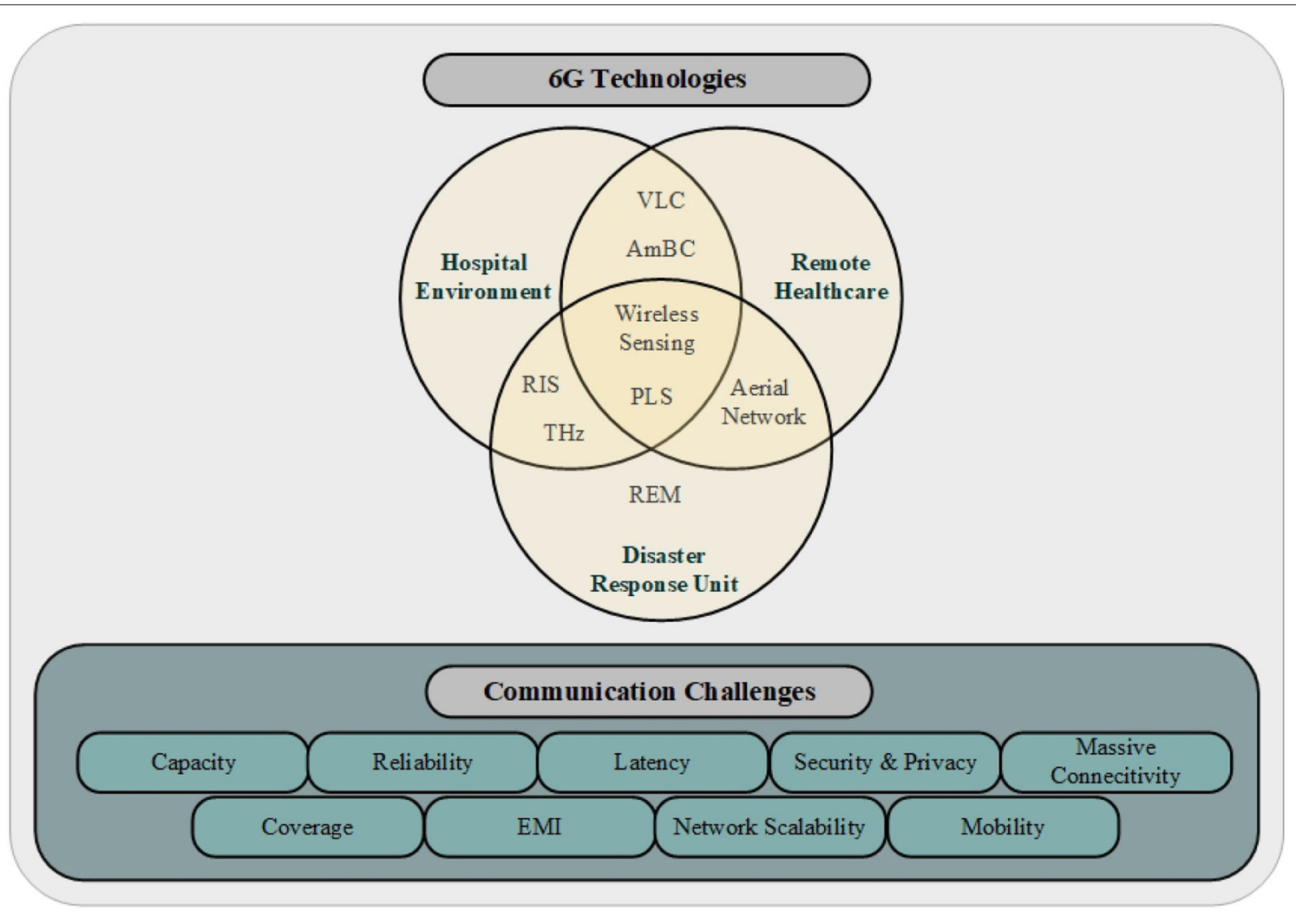

FIGURE 2 | Technologies for healthcare system challenges under 6G vision.

\subsubsection{Coverage Enhancement}

Seamless coverage and connectivity is required in all environments in healthcare systems due to diverse reasons. Network coverage in hospital is limited because of complex physical structure and sensitive devices. Currently, Wi-Fi networks are deployed for communication within the hospital. However, severe EMI issues restrict coverage in sensitive areas. In order to provide coverage in such areas, a hybrid VLC-RF communication network can be designed (Ding et al., 2015). RIS can also be deployed at certain places i.e., walls, ceiling, etc., in the hospitals for enhancing coverage as well as protecting RF restricted areas (Basar et al., 2019; Zegrar et al., 2020).

Major coverage issues related to remote health exist in rural areas, where the network is not available or the QoS is poor. Consequently, no coverage means no connectivity, thus, leaving a large population with no tele-healthcare facilities. Similar problems of coverage are found in field hospitals. In this aspect, aerial networks are one of the strongest candidates for coverage enhancement, particularly the UAVs, HAPs, and satellites (Zhou et al., 2020). These networks can enhance the coverage by acting as base stations or relaying nodes (Rinaldi et al., 2020; Saarnisaari et al., 2020). Other ways to achieve the coverage enhancement include large scale ad-hoc networks, device-to-device (D2D) communication, and mobile base stations. However, NTNs have better coverage compared to other networks. Therefore, hybrid aerial-terrestrial networks can be used to provide flexibility and network scalability for disaster scenarios when the network infrastructure is collapsed (Duranay and Arslan, 2019; Li et al., 2019).

\subsubsection{Reliable and Low Latency Communication Enhancement}

In healthcare systems, continuous communication with low latency and high reliability is vital for remote health and disaster response related services. For instance, tactile-based or autonomous surgery through robots using AI and machine learning $(\mathrm{ML})$ will require extremely reliable and low latency communication in the near future. Therefore, moving the services toward the edge within the vicinity of patients and utilizing the edge intelligence can reduce the latency and increase the reliability of the communication link (Nayak and Patgiri, 2020). Smart ambulances containing medical equipment require extremely reliable and low latency communication to communicate with medical staff at hospitals for real time monitoring and sensitive data transfer, like ECG and EEG. For safe self-driving of smart ambulances, a relatively new concept called joint radar communication (JRC) can be used to enable reliable sensing and V2X communication (Ma et al., 2020). Edge intelligence can also be used for autonomous ambulances and teleoperated vehicles to support very high mobility. Furthermore, mobility enhancement can be achieved through NTN platforms with their large coverage area. Moreover, 


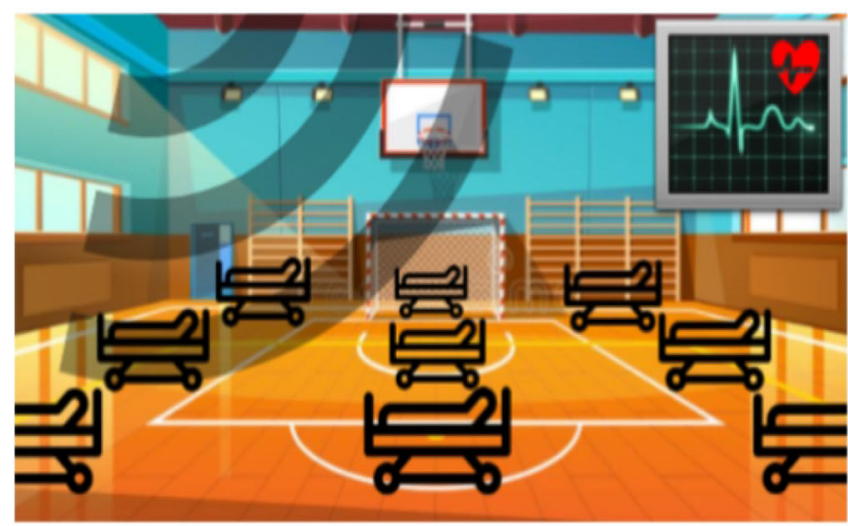

A

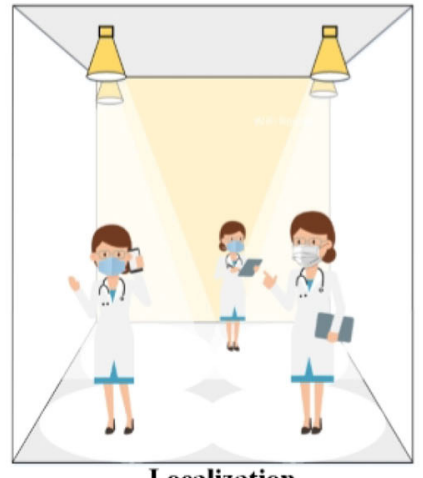

Localization

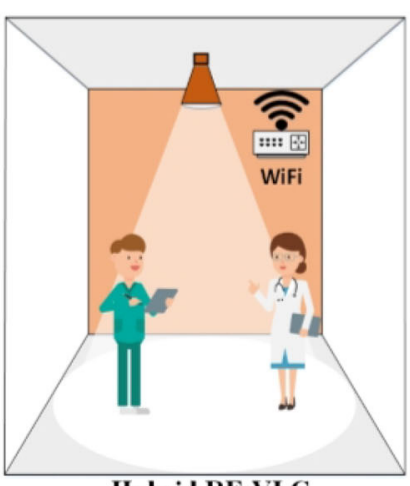

Hybrid RF-VLC

C

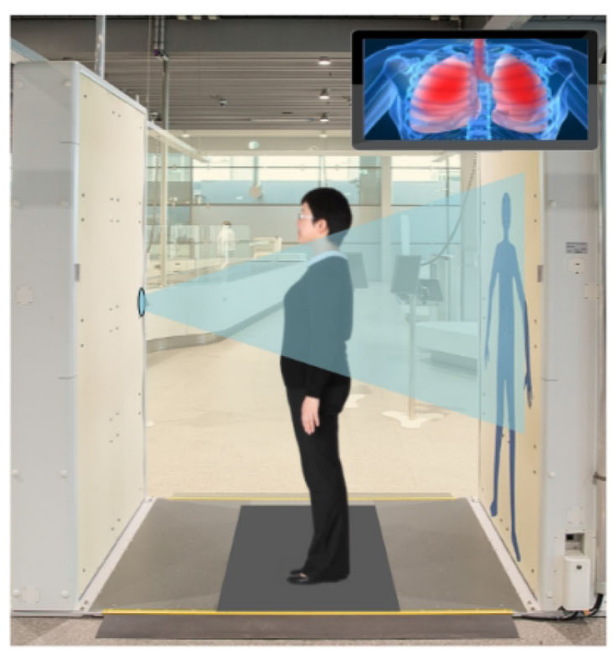

B

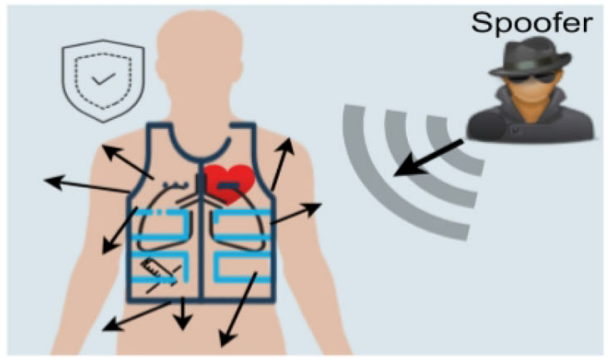

D

FIGURE 3 | Implementation of 6G technologies in healthcare system, (A) Wireless sensing in field hospital, (B) THz for COVID-19 screening, (C) VLC for localization and capacity enhancement, (D) Smart vest for WBAN security.

handover issues (i.e., delay jitter) due to mobility of patient with implanted medical devices can be addressed through the applications of radio environment mapping (REM) and AI assisted cognitive radios. In addition, utilizing the diversity in hybrid RF-VLC, and terrestrial-aerial networks can achieve the required reliable and low latency communication in indoor and outdoor environments, respectively.

\subsubsection{Sensing and Communication Enhancement}

During the COVID-19 outbreak, some countries have faced long lines and crowding outside hospitals due to lack of medical facilities and resources. Health monitoring through IoMT devices can possibly reduce this load. However, it faces two main issues; massive connectivity and shortage of IoMT devices. WiFi sensing can be helpful in these circumstances for health monitoring and patient tracking (Adib et al., 2015). Additionally, it can improve the rapid response by eliminating the disinfection process of reusable medical equipment and removing the battery limitations of IoMT devices. Similar strategies can be applied in the field hospital, where there is no existing medical facilities available for health monitoring as shown in Figure 3A. Furthermore, position and location can also be acquired through Wi-Fi sensing (Hsu et al., 2019), which maybe required in a large place with lots of patients. JRC can also be deployed to measure the environment and get the data for accurate positioning at high carrier frequencies.

Another application of wireless sensing is identifying crowding in indoor environments through Wi-Fi signals for maintaining social distancing in COVID-19. In outdoors, a more generalized concept of sensing called REM can give the better information about social distancing implementations. In addition, REM through tele-operated vehicles and drones can also used for surveillance and monitoring the social distancing violations (Turkmen et al., 2020). Moreover, sensor-based abnormal activity detection can be studied to pre-event indicators of disaster scenarios (Yin et al., 2008). AI and ML assisted REM can also be utilized in a similar manner for early awareness, initiating the rapid response and getting the insight of scale and location of the disaster. Sensing technologies can also be used for the non-invasive detection of the COVID-19. One way to do that is terahertz imaging, in which the terahertz beams are directed over the chest of the person to take the images that show the Doppler effect of terahertz waves as shown in Figure 3B (Taylor et al., 2020). These images can be classified using advanced deep learning and AI algorithms to identify the infected and healthy cells based on water content. Unlike other 
screening technologies, $\mathrm{THz}$ has no harmful effects on the living cells (Rothbart et al., 2019).

\subsubsection{Accurate Positioning and Localization}

Indoor and outdoor positioning can be of significant importance to provide a rapid response during the COVID-19 outbreak. Outdoor positioning and localization can be achieved through the global positioning system (GPS). However, GPS cannot provide accurate indoor positioning due to the complex electromagnetic propagation environment (Dang et al., 2020). When the hospitals become overcrowded with patients in a disaster situation, it becomes more complicated to locate the doctors, medical staff, and patients. Locating the medical staff and patients for real-time coordination between patients and medical staff and timely response to critical patients can be performed through wireless fidelity (Wi-Fi) real-time positioning system. However, in sensitive areas where Wi-Fi access points are not available, VLC access points can be installed. Due to the small coverage area of VLC access points, accurate position and location can be identified as shown in Figure 3C. Other applications of positioning and localization include medical robotics, elderly people activities, and remote surgery. Particularly in remote surgery, highly accurate localization and positioning are required. Such a high level of accuracy can be achieved through $\mathrm{THz}$ technology with simultaneous localization and mapping based techniques (Sarieddeen et al., 2020; Xiao and Zeng, 2020). A hybrid THz and VLC system can also be designed for achieving very accurate positioning and localization.

\subsubsection{Ensuring Security and Privacy}

Healthcare technologies expected in $6 \mathrm{G}$ should be robust to any security and privacy attacks. For instance, end-to-end link security is mandatory during the remote surgery because any attack can alter the information going to the robotic apparatus and threaten the patient's life. Cryptography based techniques are currently being used for security and privacy concerns of healthcare system. However, these algorithms have high complexity. With the development of quantum cryptography and low complexity algorithms, highly secure communication can be designed for protection (Nawaz et al., 2019). Applying quantum random number generators and quantum key distribution protocols can ensure the security in healthcare systems.

Aside from cryptography based techniques, physical layer security have been developed to make the communication link secure from jamming, spoofing and eavesdropping. PLS techniques, including channel based adaptation and key generation, cross layer security etc., can be applied to improve the communication security (Hamamreh et al., 2019; Naderi et al., 2020). In healthcare services, these techniques are of significant importance due to the low computational complexity in ultralow power communication technologies. For example, external devices can be used to protect the WBAN and IoBNT from eavesdropping by simultaneously receiving and jamming the information signals at locations other than that of the intended receiver. Channel and hardware based authentication techniques can also be used to avoid unauthorized access to the patient's critical information (Furqan et al., 2019). Moreover, the privacy of the patients can be protected through edge technologies by filtering the important data before sending to the cloud. In order to achieve this, AI based edge technologies can be used for distinguishing medical data before transmission and storing the private information in the local memory. Another way to protect the confidentiality of the data is deploying BC technology in the healthcare systems. BC will ensure the privacy and security of the patient's critical information by trustworthy data sharing through digital and distributed ledgers rather than a centralized database. Furthermore, in WBAN control unit and medical devices exchange sensitive messages and medical information of patients, any security breach can invade the privacy or cause unwanted actions. In this case, smart vest/cloak integrated advanced RIS technology called reconfigurable meta-surfaces can be a fascinating solution for securing WBAN communication, which is able to filter and manipulate the signals against jamming and spoofing attacks as shown in Figure 3D (Yang et al., 2016; Di Renzo et al., 2019).

\subsection{G Implementation Challenges}

6G technologies are still under discussion and several candidate technologies have been proposed in this paper to solve the healthcare challenges. However, the challenges related to the implementation of these technologies are yet to be defined. The first and the foremost challenge is backward compatibility, which means how to make the $6 \mathrm{G}$ compatible with the technologies of the earlier generations. For instance, utilization of higher frequency bands i.e., $\mathrm{THz}$ and VLC need new transceiver design and network architecture due to high propagation losses and small coverage areas, respectively. Thus, it is a challenging task to make these technologies backward compatible. Additionally, the dense small cell deployment will increase the cost and complexities. Another major concern regarding the use of higher frequencies is potential health issues such as damage to the skin or eye tissues. Therefore, health standards must be followed while developing these technologies for the safety and healthcare of users. Moreover, energy efficiency is critical for $6 \mathrm{G}$ technologies especially for UAVs, where efficient energy harvesting and utilization protocols are needed to support the longevity of these technologies under disaster scenarios when the energy resources are limited. Lastly, most of the technologies are going through either development or standardization stages, until the standardization and deployment, real implementation problems cannot be defined.

\section{DISCUSSION}

Increasing number of pandemic and other disaster situations necessitate the advancements in communication technologies to cope with the current and future health crisis. In this regard, challenges and weaknesses of healthcare systems from a communication perspective are discussed under three categories i.e, hospital environment, remote healthcare and disaster response unit. Solutions to these challenges are provided through $6 \mathrm{G}$ technologies that are being studied with a humanitarian perspective to serve the people in all environments. The future $6 \mathrm{G}$ communication systems should be designed to support 
healthcare systems with advanced communication technologies during pandemic and disaster scenarios. $6 \mathrm{G}$ supported healthcare communication can minimize the adverse effects and provide the rapid response in future pandemic situations. In particular, IoMT, VLC, wireless sensing, PLS, and autonomous vehicles will revolutionize the healthcare system. There are some other technologies e.g., waveform, which are not discussed yet under the $6 \mathrm{G}$ visions but can help in healthcare communication to solve several challenges such as capacity, security, reliability etc. Furthermore, medical technologies are also developing rapidly from wearable IoMT to implantable IoBNT and neuralink to understand the brain activities for improving the quality of life. In pandemic outbreaks, these technologies will not only provide the regular healthcare remotely, but will also assist in early diagnosis. However, a precise integration of these technologies into the healthcare system for a real-time implementation is still an open challenge. In addition, dealing with psychological effects that arise during lock down needs to be studied from the perspective of communication technologies.

\section{REFERENCES}

Adib, F., Mao, H., Kabelac, Z., Katabi, D., and Miller, R. C. (2015). "Smart homes that monitor breathing and heart rate," in Proceedings of the 33rd Annual ACM Conference on Human Factors in Computing Systems, CHI '15 (New York, NY: Association for Computing Machinery), 837-846. doi: 10.1145/2702123.2702200

Ahad, A., Tahir, M., and Yau, K.-L. A. (2019). 5G-based smart healthcare network: architecture, taxonomy, challenges and future research directions. IEEE Access 7, 100747-100762. doi: 10.1109/ACCESS.2019.2930628

Ahmed, I., Karvonen, H., Kumpuniemi, T., and Katz, M. (2020). Wireless communications for the hospital of the future: requirements, challenges and solutions. Int. J. Wireless Inform. Netw. 27, 4-17. doi: 10.1007/s10776-019-00468-1

Akyildiz, I. F., Kak, A., and Nie, S. (2020). 6G and beyond: the future of wireless communications systems. IEEE Access 8, 133995-134030. doi: 10.1109/ACCESS.2020.3010896

Basar, E., Di Renzo, M., De Rosny, J., Debbah, M., Alouini, M.-S., and Zhang, R. (2019). Wireless communications through reconfigurable intelligent surfaces. IEEE Access 7, 116753-116773. doi: 10.1109/ACCESS.2019.2935192

Berardinelli, G., Mogensen, P., and Adeogun, R. O. (2020). "6G subnetworks for life-critical communication," in 2020 2nd $6 \mathrm{G}$ Wireless Summit (6G SUMMIT) (Levi: IEEE), 1-5. doi: 10.1109/6GSUMMIT49458.2020.9083877

Boyle, J. (2006). Wireless technologies and patient safety in hospitals. Telemed. J. eHealth 12, 373-382. doi: 10.1089/tmj.2006.12.373

Chamola, V., Hassija, V., Gupta, V., and Guizani, M. (2020). A comprehensive review of the COVID-19 pandemic and the role of IoT, drones, AI, blockchain, and 5G in managing its impact. IEEE Access 8, 90225-90265. doi: 10.1109/ACCESS.2020.2992341

Cook, D. J., Duncan, G., Sprint, G., and Fritz, R. L. (2018). Using smart city technology to make healthcare smarter. Proc. IEEE 106, 708-722. doi: 10.1109/JPROC.2017.2787688

Coronese, M., Lamperti, F., Keller, K., Chiaromonte, F., and Roventini, A. (2019). Evidence for sharp increase in the economic damages of extreme natural disasters. Proc. Natl. Acad. Sci. U.S.A. 116, 21450-21455. doi: 10.1073/pnas.1907826116

Da Costa Daniel Benevides, Y. H.-C. (2020). Grand challenges in wireless communications. Front. Commun. Netw. 1:1. doi: 10.3389/frcmn.2020.00001

Dang, S., Amin, O., Shihada, B., and Alouini, M.-S. (2020). What should 6G be? Nat. Electron. 3, 20-29. doi: 10.1038/s41928-019-0355-6

Di Renzo, M., Debbah, M., Phan-Huy, D.-T., Zappone, A., Alouini, M.-S., Yuen, C., et al. (2019). Smart radio environments empowered by reconfigurable AI
Finally, significant efforts are required to develop a fully equipped healthcare system that can offer all necessary services for disaster scenarios. As being wireless communication engineers, we are uniquely able to leverage our research strengths to quickly respond to the current COVID-19 crisis, as well as future pandemics.

\section{DATA AVAILABILITY STATEMENT}

The original contributions presented in the study are included in the article/supplementary material, further inquiries can be directed to the corresponding author/s.

\section{AUTHOR CONTRIBUTIONS}

This work was developed with the collaboration of all authors. MBJ and AED wrote and revised the manuscript under HA's supervision. HA also reviewed the manuscript. All authors have read and approved the published version of the manuscript.

meta-surfaces: an idea whose time has come. EURASIP J. Wireless Commun. Network. 2019, 1-20. doi: 10.1186/s13638-019-1438-9

Ding, W., Yang, F., Yang, H., Wang, J., Wang, X., Zhang, X., et al. (2015). A hybrid power line and visible light communication system for indoor hospital applications. Comput. Indus. 68, 170-178. doi: 10.1016/j.compind.2015.01.006

Duranay, A. E., and Arslan, H. (2019). "The evaluation of FFR for interference management in coordinated hybrid terrestrial-aerial network," in 2019 IEEE 30th Annual International Symposium on Personal, Indoor and Mobile Radio Communications (PIMRC) (İstanbul), 1-6. doi: 10.1109/PIMRC.2019.8904259

Elayan, H., Amin, O., Shihada, B., Shubair, R. M., and Alouini, M. (2020). Terahertz band: the last piece of RF spectrum puzzle for communication systems. IEEE Open J. Commun. Soc. 1, 1-32. doi: 10.1109/OJCOMS.2019.29 53633

Fieux, M., Duret, S., Bawazeer, N., Denoix, L., Zaouche, S., and Tringali, S. (2020). Telemedicine for ENT: effect on quality of care during COVID19 pandemic. Eur. Ann. Otorhinolaryngol. Head Neck Dis. 137, 257-261. doi: 10.1016/j.anorl.2020.06.014

Furqan, H. M., Solaija, M. S. J., and Arslan, H. (2019). Intelligent physical layer security approach for V2X communication. arXiv preprint arXiv:1905.05075.

Hamamreh, J. M., Furqan, H. M., and Arslan, H. (2019). Classifications and applications of physical layer security techniques for confidentiality: a comprehensive survey. IEEE Commun. Surv. Tutorials 21, 1773-1828. doi: 10.1109/COMST.2018.2878035

Hassija, V., Chamola, V., Saxena, V., Jain, D., Goyal, P., and Sikdar, B. (2019). A survey on IoT security: application areas, security threats, and solution architectures. IEEE Access 7, 82721-82743. doi: 10.1109/ACCESS.2019. 2924045

Hsu, C.-Y., Hristov, R., Lee, G.-H., Zhao, M., and Katabi, D. (2019). "Enabling identification and behavioral sensing in homes using radio reflections," in CHI '19 (New York, NY: Association for Computing Machinery), 1-13. doi: 10.1145/3290605.3300778

Janjua, M. B., da Costa, D. B., and Arslan, H. (2020). User pairing and power allocation strategies for 3D VLC-NOMA systems. IEEE Wireless Commun. Lett. 9, 866-870. doi: 10.1109/LWC.2020.2973628

Li, B., Fei, Z., and Zhang, Y. (2019). UAV communications for $5 \mathrm{G}$ and beyond: Recent advances and future trends. IEEE Internet Things J. 6, 2241-2263. doi: 10.1109/JIOT.2018.2887086

Li, D. (2019). 5G and intelligence medicine-how the next generation of wireless technology will reconstruct healthcare? Precis. Clin. Med. 2, 205-208. doi: 10.1093/pcmedi/pbz020

Ma, D., Shlezinger, N., Huang, T., Liu, Y., and Eldar, Y. C. (2020). Joint radar-communication strategies for autonomous vehicles: combining 
two key automotive technologies. IEEE Signal Process. Mag. 37, 85-97. doi: 10.1109/MSP.2020.2983832

Matinmikko-Blue, M., Aalto, S., Asghar, M. I., Berndt, H., Chen, Y., Dixit, S., et al. (2020). White paper on 6G drivers and the UN SDGs. arXiv preprint arXiv:2004.14695.

Naderi, S., da Costa, D. B., and Arslan, H. (2020). Joint random subcarrier selection and channel-based artificial signal design aided PLS. IEEE Wireless Commun. Lett. 9, 976-980. doi: 10.1109/LWC.2020.2976979

Nawaz, S. J., Sharma, S. K., Wyne, S., Patwary, M. N., and Asaduzzaman, M. (2019). Quantum machine learning for 6G communication networks: State-of-the-art and vision for the future. IEEE Access 7, 46317-46350. doi: 10.1109/ACCESS.2019.2909490

Nayak, S., and Patgiri, R. (2020). 6G communication technology: a vision on intelligent healthcare. arXiv preprint arXiv:2005.07532. doi: 10.4108/eai.17-8-2020.166293

Ohannessian, R., Duong, T. A., and Odone, A. (2020). Global telemedicine implementation and integration within health systems to fight the COVID19 pandemic: a call to action. JMIR Public Health Surveill. 6:e18810. doi: $10.2196 / 18810$

Pramanik, P. K., Nayyar, A., and Pareek, G. (2019). “Chapter 7 - WBAN: driving e-healthcare beyond telemedicine to remote health monitoring: architecture and protocols," in Telemedicine Technologies, eds H. D. Jude and V. E. Balas (Academic Press), 89-119. doi: 10.1016/B978-0-12-816948-3.00007-6

Rahman, M. A., Hossain, M. S., Alrajeh, N. A., and Guizani, N. (2020). B5G and explainable deep learning assisted healthcare vertical at the edge: COVID-19 perspective. IEEE Netw. 34, 98-105. doi: 10.1109/MNET.011.2000353

Rinaldi, F., Maattanen, H.-L., Torsner, J., Pizzi, S., Andreev, S., Iera, A., et al. (2020). Non-terrestrial networks in 5G \& beyond: a survey. IEEE Access 8, 165178-165200. doi: 10.1109/ACCESS.2020.3022981

Rothbart, N., Holz, O., Koczulla, R., Schmalz, K., and Hübers, H.-W. (2019). Analysis of human breath by millimeter-wave/terahertz spectroscopy. Sensors 19:2719. doi: 10.3390/s19122719

Saarnisaari, H., Dixit, S., Alouini, M.-S., Chaoub, A., Giordani, M., Kliks, A., et al. (2020). A 6G white paper on connectivity for remote areas. arXiv preprint arXiv:2004.14699.

Saeed, N., Bader, A., Al-Naffouri, T. Y., and Alouini, M.-S. (2020). When wireless communication responds to covid-19: combating the pandemic and saving the economy. Front. Commun. Netw. 1:3. doi: 10.3389/frcmn.2020.566853

Sarieddeen, H., Saeed, N., Al-Naffouri, T. Y., and Alouini, M.-S. (2020). Next generation terahertz communications: a rendezvous of sensing, imaging, and localization. IEEE Commun. Mag. 58, 69-75. doi: 10.1109/MCOM.001.1900698

Tanwar, S., Parekh, K., and Evans, R. (2020). Blockchain-based electronic healthcare record system for healthcare 4.0 applications. J. Inform. Secur. Appl. 50:102407. doi: 10.1016/j.jisa.2019.102407

Taylor, W., Abbasi, Q. H., Dashtipour, K., Ansari, S., Shah, S. A., Khalid, A., et al. (2020). A review of the state of the art in non-contact sensing for COVID-19. Sensors 20:5665. doi: 10.3390/s20195665

Tian, S., Yang, W., Le Grange, J. M., Wang, P., Huang, W., and Ye, Z. (2019). Smart healthcare: making medical care more intelligent. Glob. Health J. 3, 62-65. doi: 10.1016/j.glohj.2019.07.001
Turkcell (2020). Turkcell group. Our Initial Take on COVID-19.

Turkmen, H., Solaija, M. S. J., Furqan, H. M., and Arslan, H. (2020). Generalized radio environment monitoring for next generation wireless networks. arXiv.

Van Huynh, N., Hoang, D. T., Lu, X., Niyato, D., Wang, P., and Kim, D. I. (2018). Ambient backscatter communications: a contemporary survey. IEEE Commun. Surv. Tutorials 20, 2889-2922. doi: 10.1109/COMST.2018.2841964

Vora, J., Italiya, P., Tanwar, S., Tyagi, S., Kumar, N., Obaidat, M. S., et al. (2018). "Ensuring privacy and security in e-health records," in 2018 International Conference on Computer, Information and Telecommunication Systems (CITS) (Colmar: IEEE), 1-5. doi: 10.1109/CITS.2018.8440164

Wei, K., Zhang, L., Guo, Y., and Jiang, X. (2020). Health monitoring based on internet of medical things: architecture, enabling technologies, and applications. IEEE Access 8, 27468-27478. doi: 10.1109/ACCESS.2020.2971654

Wijesooriya, N. R., Mishra, V., Brand, P. L., and Rubin, B. K. (2020). COVID-19 and telehealth, education, and research adaptations. Paediatr. Respirat. Rev. 35, 38-42. doi: 10.1016/j.prrv.2020.06.009

Willan, J., and King, A. J., Jeffery, K., and Bienz, N. (2020). Challenges for NHS hospitals during covid-19 epidemic. BMJ. 368:m1117. doi: 10.1136/bmj.m1117

Xiao, Z., and Zeng, Y. (2020). An overview on integrated localization and communication towards 6G. arXiv preprint arXiv:2006.01535.

Yaacoub, E., and Alouini, M.-S. (2020). A key 6G challenge and opportunityconnecting the base of the pyramid: a survey on rural connectivity. Proc. IEEE 108, 533-582. doi: 10.1109/JPROC.2020.2976703

Yang, H., Cao, X., Yang, F., Gao, J., Xu, S., Li, M., et al. (2016). A programmable metasurface with dynamic polarization, scattering and focusing control. Sci. Rep. 6:35692. doi: 10.1038/srep35692

Yin, J., Yang, Q., and Pan, J. J. (2008). Sensor-based abnormal humanactivity detection. IEEE Trans. Knowledge Data Eng. 20, 1082-1090. doi: 10.1109/TKDE.2007.1042

Zarkeshev, A., and Csiszár, C. (2019). Are people ready to entrust their safety to an autonomous ambulance as an alternative and more sustainable transportation mode? Sustainability 11:5595. doi: 10.3390/su11205595

Zegrar, S. E., Afeef, L., and Arslan, H. (2020). A general framework for RISaided mmWave communication networks: channel estimation and mobile user tracking. arXiv.

Zhou, J., Yang, J., and Lu, L. (2020). "Research on multi-UAV networks in disaster emergency communication," in IOP Conference Series: Materials Science and Engineering, Vol. 719 (Wuhan: IOP Publishing), 012054.

Conflict of Interest: The authors declare that the research was conducted in the absence of any commercial or financial relationships that could be construed as a potential conflict of interest.

Copyright (c) 2020 Janjua, Duranay and Arslan. This is an open-access article distributed under the terms of the Creative Commons Attribution License (CC BY). The use, distribution or reproduction in other forums is permitted, provided the original author(s) and the copyright owner(s) are credited and that the original publication in this journal is cited, in accordance with accepted academic practice. No use, distribution or reproduction is permitted which does not comply with these terms. 